\title{
Synchronized Dancing of an Industrial Manipulator and Humans with Arbitrary Music
}

\author{
Figen Özen ${ }^{1}$, Dilek Bilgin Tükel ${ }^{2}$, Georgi Dimirovski ${ }^{2}$
}

${ }^{1}$ Haliç University, Department of Electrical and Electronics Engineering, Sütlüce Mah, Nr 82, İmrahor Cad, 34445, Istanbul, Turkey, figenozen@ halic.edu.tr

${ }^{2}$ Doğuş University, Department of Control and Automation Engineering, Hasanpaşa Mah, Zeamet Sok, Nr 21, 34722, Istanbul, Turkey

dtukel@dogus.edu.tr,gdimirovski@dogus.edu.tr

Abstract: An extended Labanotation for an industrial robot was developed and applied. A user-friendly program was designed to help design dance choreography. The program, LabanRobot, has a simple interface that can be used without any prior knowledge of the robot. The choreographer should keep geometry in mind and plan the dance sequence accordingly. The conversion of dance sequences to robot motion is done automatically by the program. Details of the algorithm are given. Examples of simulation and on-stageperformance are shown.

Keywords: industrial robot; Labanotation; dance

\section{Introduction}

Since the beginning of human existence, the environment and life itself has posed many difficult problems for humans. The strength of humans was limited and insufficient to achieve the many tasks that life on earth required. Humans may have been physically weak, but their imagination and intelligence were strong. To overcome their physical limitations, they imagined artificial humans to help. The dream of artificial servants dates back to before Christ. Aristotle said, "If every tool, when ordered, or even of its own accord, could do the work that befits it, then there would be no need either of apprentices for the master workers or of slaves for the lords." He imagined such a machine even when humanity was far from realizing this dream. Eventually, automata, which can do some humble work for people, were finally created. But they were far from satisfying human imagination. Research and attempts to design better ones accelerated and goals became even more ambitious when more tools were available. 
Today, in the age of Industry 4.0, millions of robots are in use. Research continues to build more effective industrial robots to achieve more sophisticated tasks as part of the smart factory concept, where robots act as cyberphysical systems. They communicate with other robots and humans through an Internet of Things (IoT). Human-robot collaboration and this is a challenge, since industrial robots may accidentally harm humans. There has been a lack of sensors that produce data similar to the human senses and a shortage of intelligent control algorithms to avoid accidents during collaboration.

In this work, an industrial robot arm was programmed to perform a popular dance. A simple, user-friendly graphical interface was created to aid inputting the choreography. The user does not have to be a programmer to design a dance. Color codes and simple buttons are used to simplify the design process. Section 2 discusses the notations of movement and dance. Section 3 gives a brief review of the literature. Section 4 describes the extended Labanotation proposed in this paper and its application with a robot. Section 5 draws conclusions and indicates further research topics.

\section{Expressing Robot Motion}

Robot motion has been a research topic for decades. When it comes to humanoids, movement is expected to be human-like, but modeling human motion is a very complex task. After designing a humanoid, then the inevitable problem arises: How should one tell the robot to behave like a human?

If a human has to open a door, she or he goes to the door and opens it. This task usually requires no deep thinking for a human. On the other hand, if a humanoid is made to open the door, then the task must be split into a sequence of sub-tasks. For example, locating the door handle, raising the arm, grasping the handle with the hand, pushing the handle, pulling the door, releasing the handle, and stopping.

All the sub-tasks must be translated into a language that allows the robot to perform them correctly. This takes the sub-task into another domain, where the application of control results in action. For example, the sub-task of raising the arm must translated to torque at specific angles by certain actuators in the joints.

\subsection{Translation of Sub-Tasks into Machine Language}

To translate sub-tasks into machine language, various notation systems can be used. There are around ninety systems but only a few are used in robotics. The most popular ones are Labanotation, Benesh notation, and Eshkol Wachman notation, in order of popularity. They have been borrowed from the field of dance, but are not limited to it. They have also been used in gymnastics, mime, circus 
performance, anthropology, ergonomics, neurology, clinical research, animation, and human-computer interaction [1].

Due to the complexity of movement, there has been great interest in representing it in simpler terms. There have been many attempts to codify movement, especially in the field of dance. In dance education, the traditional method is supervised learning, where a teacher shows a student how to dance a given pattern. This requires extended one-on-one study, is not very efficient, and relies too much on the student's memory. The dance notation systems strived for efficiency and reliability.

Dance notations are done with many different forms such as letters, numbers, stick figures, and realistic figures. There have been attempts to represent robot movement with dance notation, especially humanoids. There is no notation that can adequately express every possible movement. This leaves the roboticist with fewer options. The majority of the work employing movement notation in the robotics uses Labanotation.

In the following section, the Laban, Benesh and Eshkol Wachman notation systems will be reviewed, since they are by far the most common ones. All three notation systems were developed in the twentieth century. They require a considerable amount of time to learn. Even though they share the same aim, their approaches are different.

\subsubsection{Laban Notation}

Laban notation was devised by the dancer and the choreographer Rudolph Laban. It is also called Kinetography Laban and Labanotation, which was coined by Ann Hutchinson Guest and has gained popularity. Kinetography Laban presents a compact view of the body.

In Laban notation, successive movements are shown vertically and simultaneous movements are shown horizontally. The duration of each movement is indicated by vertical length. The part of the body involved is also expressed vertically [2].

The Laban system describes a movement in terms of the following elements:
a) Body
b) Space
c) Effort
d) Shape

The first element, body, identifies which body parts are involved. The 'space' element indicates the space and extent of the movement. Here, the Kinesphere, the term coined by Rudolph Laban, is to be highlighted. It refers to the volume created and occupied by a human. Depending on the movement, it can become larger or smaller [3]. The 'effort' element refers to the energy involved. The 'shape' element is the form of the body and its evolution during the movement. 
Laban notation is very detailed. There is also an abbreviated representation, Laban motif notation. This was devised by Ann Hutchinson Guest and others to use in dance education, and focuses on what is different or similar. Especially when the repetitive patterns are involved, it saves time and energy [4].

Labanotation uses a staff, similar to a music score. Unlike a music score, Labanotation is read vertically from bottom to top. It is divided into columns to represent the body parts (Fig. 1). The centerline represents the vertical center of the body. The left side of the staff represents the left side of the body, and the right side of the staff, the right side of the body.

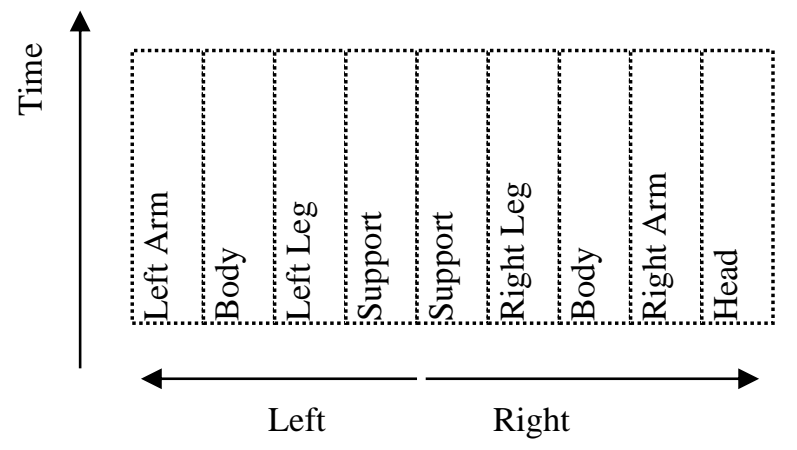

Figure 1

Organization of the staff of a Laban score

Labanotation has symbols for nine different directions, which can be high, medium or low, so there are 27 possible combinations. Fig. 2 shows the direction symbols and position marks. In the figure, the shape of the symbol indicates the direction, shading, and the level or height of the movement.

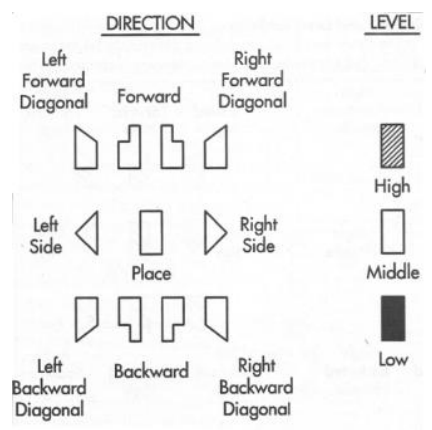

Figure 2

Laban symbols

An example of Laban notation is given in Fig. 3. 


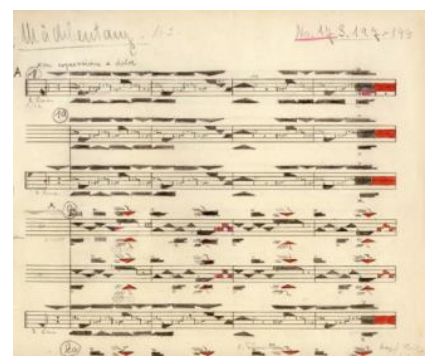

Figure 3

Example of Labanotation

Among Labanotation programs, LabanWriter is the most widely used. The program was developed at the Ohio State University. It prepares Labanotation scores and digitizes them [5].

Laban movement analysis is based on Rudolph Laban's work but it deals with dynamics and effects that Labanotation does not include.

The Effort Shape notation was also devised by Laban, but it is less popular. It includes the following parameters:
a) Weight (light, strong)
b) Space (indirect, direct)
c) Flow (free, bound)
d) Time (sustained, quick) [6]

Here, time refers to rhythm, tempo, phasing, and duration of the movement. Weight refers to the movement's softness, smoothness, sharpness, and energy. Flow is about the combination of variation of movements, using recurring elements, contrast, and repetition. Basic types of movements are dabbing, gliding, floating, flicking, thrusting, pressing, wringing, and slashing [7].

\subsubsection{Benesh Notation}

Benesh notation was devised by Rudolph Benesh and Joan Rothwell in the midtwentieth century. It uses key frames. It is drawn on a staff, like Laban notation, but sequences are written horizontally from left to right. The staff has five vertical lines, each for different body parts. Stronger movements are indicated by a bolder drawing. Fig. 4 shows an example of Benesh notation.

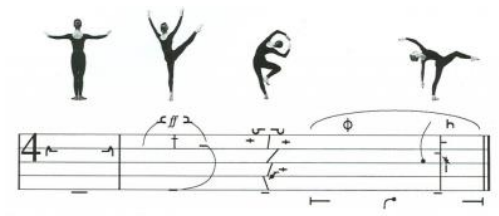

Figure 4

Example of Benesh notation 


\subsubsection{Eshkol Wachman Notation}

Eshkol Wachman notation was devised by the dancer and choreographer Noa Eshkol and the architect Abraham Wachman in the 1960s. In this notation, the body is viewed as a composition of articulated limbs, which can lie between two joints or be connected to a joint at only one end. It is recorded in a matrix, not a staff. The left side of the matrix indicates which body parts move. Sequences are written from left to right and the end of the movement is shown by a bold bar [8]. Fig. 5 shows an example.

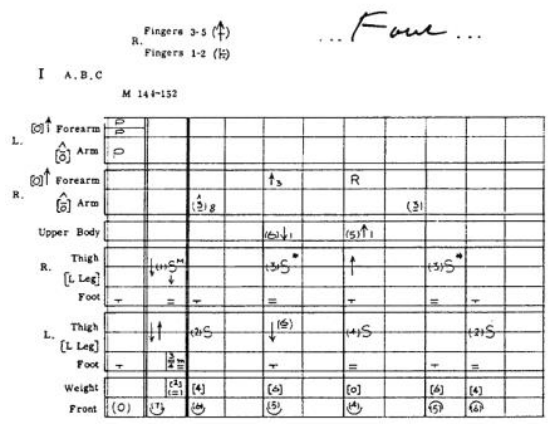

Figure 5

Example of Eshkol Wachman notation

\section{Literature Review}

Research on dance in the robotics field deals with many problems, which can be grouped as motion capture, processing the captured images/videos, processing music to dance with, representing the required motion using dance notation, converting the notation into inputs for the joints of a robot, and evaluating the dance performance of a robot. Naturally, not all of these issues are dealt with in every publication.

Peng, Zhou, $\mathrm{Hu}$, Chao and $\mathrm{Li}$ [9] provide a thorough review of the robotics dance field since the 1990s. They describe the following research areas: robotic dancers that can cooperate with humans, imitate human dance, synchronize with music, and creating robotic choreography. They emphasized that cooperation between a robot and a human is harder to achieve than coordination between robots and that the imitation of human movement by a robot is still in its infancy. They conclude that synchronizing with music requires many aspects of music to be taken into consideration, but so far, only beat, tempo, and rhythm have been studied. Finally, they mention that work remains to be done on creating choreography with human aesthetic values and improvisation. 
Afsar, Cortez and Santos [10] surveyed approaches to human behavior detection from video. The steps involved are initialization, tracking, pose, and recognition. They review each step in detail and tabulate the literature to aid future research.

Most papers deal with a certain aspect of robot dance or a specific dance. The rest of this section summarizes some of the outstanding research in the field.

Hachimura and Nakamura [11] segmented motion data and quantize motion direction and duration for Labanotation. A Laban editor with limited scope was developed for dance education.

Kojima, Hachimura, and Nakamura [12] modeled the human body with 21 joints and study the speed of joint motion. Labanotation data was converted into animation data. Only basic Labanotation functions were simulated, thus compromising the smoothness of the motion. An interface for LabanEditor was introduced. Interaction with the environment was not taken into account.

Nakamura, Niwayama, Tabata, and Kumo [13] developed a dance training system consisting of a motion capture system, a projector for projecting the teacher's footprints on the floor, and a robot acting as a mobile mirror. Two traditional Japanese dances were used for testing. The mirror robot had to position itself at a suitable distance as the student rehearses. Since the student cannot look at the mirror robot and the teacher's footprints at the same time, the reasoning has to be modified.

Suzuki and Hashimoto [14] combined a mobile platform with a motion interface system, a controller, and output devices to study human robot interaction with a semiautonomous robot. In response to a performer's dance, the program iDance produces sounds and moves in compliance with the shifting weight of the performer. Neural networks were used to represent emotional states.

Kosuge and Hirata designed [15] a dance robot to study human-robot coordination coupled with physical interaction. The robot has an omni-directional mobile base and it can sense the force and torque applied by its human partner. The robot was tested with the Waltz. More dance forms need to be tested.

Choi, Kim, Oh, and You [16] balanced the dancing arm of a humanoid robot with a posture and walking control scheme that utilizes the kinematic resolution method of Center of Mass (CoM).

Jeong, Seo, and Yang [17] designed a wearable interface equipped with magnetic sensors to teach human motion to a humanoid robot. Recorded motions are processed to obtain key frames. Clustering is applied to the key frames. A computer analyzes and enacts motion primitives and kinematic calculations. The system was tested using simple motions, namely drawing a heart and boxing. More complex motions have to be taken into consideration to imitate human motions effectively. 
Kekehashi, Izawa, Shirai, Nakanishi, Okada, and Inaba [18] studied the motion of hula hooping. Torso movement was analyzed and the algorithm was tested using dedicated robots. Some stability problems were encountered. Since hula hooping is simpler than dance, in general, the algorithm needs to be adapted to the challenges of dance and later to arbitrary human motion.

Dong, Wang, Wang, Yan, and Chen [19] studied the lion dance with a 19 degreesof-freedom, under actuated, multi-legged robot. Kinematics, dynamics, and stability of the lion dance were studied, which was performed by two humans. The results showed that the robot lacked the liveliness and loveliness required to perfectly imitate the lion dance.

Wang and Kosuge [20] considered the case of the Waltz. This dance has a leader and a follower. In the study, the human was the leader and robot was the follower. The human dancer's body was modeled using an inverted pendulum. The position of the leader was estimated with data from laser range finders and an extended Kalman filter. Next step estimation was done with Hidden Markov Models. Since dancing the Waltz requires haptic interaction, human arm dynamics were used in the control loop. The paper leaves the questions of role switching and rotational motions unanswered. Robustness and optimality are issues to be dealt with.

Auguliaro, Schoellig, and D'Andrea [21] used the Laban Effort Shape concepts, namely space, time, weight, and flow, in the choreography of a group of quadrocopters. Motion and music were synchronized, and collision-free flight trajectories were calculated, but the motion library is limited and audience feedback is not incorporated.

Zhou, De la Torre, and Hodgins [22] extended Kernel K Means and Spectral Clustering to partition human motion into a fixed number of classes. Testing was done on with Carnegie Mellon University Motion Capture database videos. The results show that the computational load is very heavy, and some simplifications need to be employed. In addition, the algorithm has to be sped up for real-time application.

Radac and Precup [23] classified the ways of learning from primitives as a) timescale transformation approaches, b) temporal concatenation of primitive-based approaches, and c) time-based decomposition approaches. The primitives are useful to simplify the task, which can be very complex. They suggested an iterative learning control algorithm, which does not require much prior information about the system to be controlled. They solved the trajectory tracking problem.

Wang and Zhou [24] used a method based on time-based b-spline-decomposition for a continuous and smooth trajectory tracking. The desired trajectory was decomposed online and the control input was synthesized. The application to a microscope probe resulted in better output tracking over feedback control. 
Mussa-Ivaldi and Solla [25] investigated the primitives for motion control in neurobiological systems. They studied adaptive behavior in the nervous system for learning, making use of knowledge stored in memory.

Grymin, Neas and Farhood [26] worked on motion planning in an environment with obstacles. Their approach was based on temporal concatenation of motion primitives and the Graph theory. They provided their simulation results on a hovercraft.

Okamoto, Shiratori, Kudoh, Nakaoka, and Ikeuchi [27] dealt with a cyclic Aizubandasian dance. The dance was performed by a human and was captured using a motion capture system, sampled, and then key poses were extracted and performed by a humanoid based on key poses. The human dance was decomposed by splines. The humanoid dance consisted of lower-, middle-, and upper-body movements. Each movement was split into sub-tasks to be performed by each body part. The lower-body supported the whole body, the middle-body supplied the expression of dance and maintained balance, the upper-body expressed the dance. The algorithm has not yet been applied to other dance forms to demonstrate universality.

Ramos, Mansard, Stasse, Benazeth, Hak, and Saab [28] used motion capture to form a reference movement for a humanoid dancing with a human. To generate the movements, an operational space inverse dynamics method was used, to solve the problem of following the dance figures demonstrated by a human. The problems of coordination, control, and stabilization of balance were studied; balance was given top priority over other objectives such as visibility and posture. To maintain balance, linear momentum variation was controlled by center-of-mass acceleration and angular momentum variation was controlled by proper contact forces. Due to the lack of sensor feedback, the performance of the platform fell short of expectations.

Yoshida, Shirokura, Sigiura, Sakamato, Ono, Inami, and Igarashi [29] reported an interface for an entertainment system called RoboJockey. The interface controls a mobile and a humanoid robot. Among other actions, the robots automatically perform according to music. The humanoid robot moves its knees in response to the beat. The mobile robot does not have enough actuators to move with the rhythm, thus it simply switches actions in response to the beat. The system and the interface need improvement because high level functionality, including programming loops and sensor feedback, is not supported.

Takano and Nakamura [30] represented human motion through segmentation and encoded the segments into Hidden Markov Models. Afterwards, statistical analysis tools, such as correlation matrices, were used to predict the next motion. The algorithm should be optimized and parameter tuning should be adaptive. The correlations between the parameters need to be tested. 


\section{Experimental Setup}

For the research presented here, a computer program was written to make an industrial manipulator dance together with a human. To design a robot dance similar to human dance, the required movements are expressed with extended Labanotation. The industrial manipulator is a six-degree-of-freedom Mitsubishi RV-7FL, which is geometrically similar to a human arm.

A Laban editor was designed and programmed through a special interface. The controller calculates the input torque parameters of the manipulator after the required motion data has been supplied from the interface.

To handle objects, a hand was designed for the industrial robot. The hand consists of a vacuum pad and a vacuum generator. During dance, the hand carries dance related accessories such as flowers, matador's cape, camera, etc.

The control of the robot arm is done by CR-750D, which has servo drivers and a motion controller inside. Hierarchical trajectories are generated by Matlab and RT Toolbox 2. The host computer communicates with the robot controller via Ethernet. The interconnection is shown in Fig. 6.

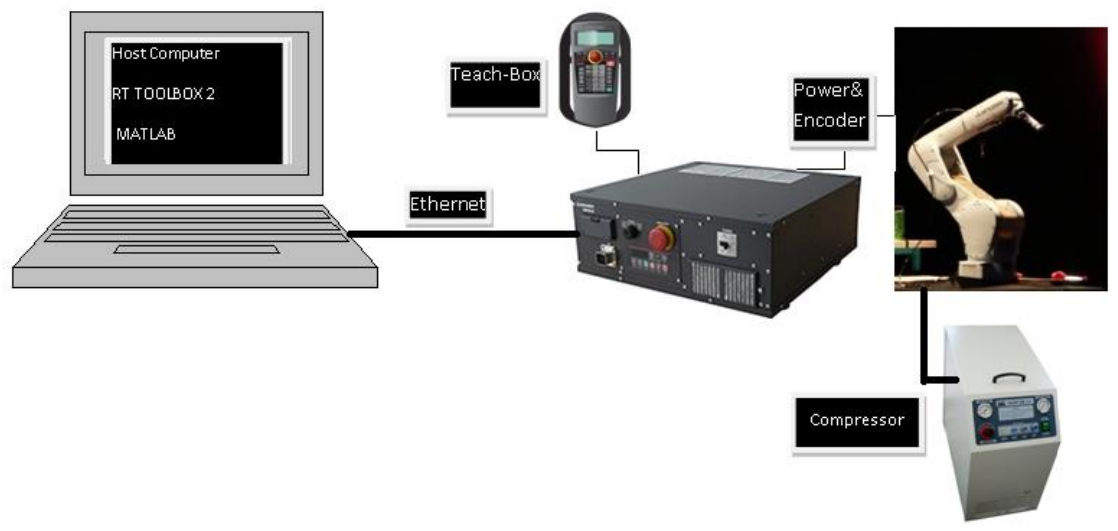

Figure 6

Mitsubishi RV-7FL robot arm with CR-750 controller and the connection of system components

To translate Labanotation into robot movement, the kinematic model of the manipulator is needed. The derivation is done and the specifications of the manipulator are summarized in Table 1.

Link lengths were obtained from the CAD data supplied by the manufacturing company [31]. The external dimensions in Fig. 7 were also supplied by the manufacturing company. 
Table 1

The specifications of the industrial robot

\begin{tabular}{|c|c|c|c|c|c|c|c|c|}
\hline Type & $\begin{array}{l}\text { Joint } \\
\text { Number }\end{array}$ & $\begin{array}{l}\text { Mitsubishi } \\
\text { RV-7FL }\end{array}$ & Type & $\begin{array}{l}\text { Joint } \\
\text { Number }\end{array}$ & $\begin{array}{l}\text { Mitsubishi } \\
\text { RV-7FL }\end{array}$ & & & \\
\hline $\begin{array}{l}\text { Degrees } \\
\text { of } \\
\text { freedom }\end{array}$ & & 6 & $\begin{array}{l}\text { Maximum } \\
\text { load } \\
\text { capacity }\end{array}$ & & $7 \mathrm{~kg}$ & & & \\
\hline $\begin{array}{l}\text { Maximum } \\
\text { reach } \\
\text { radius }\end{array}$ & & $908 \mathrm{~mm}$ & Mass & & $67 \mathrm{~kg}$ & & & \\
\hline \multirow{6}{*}{\multicolumn{4}{|c|}{ Operating range (degree) }} & 1 & \pm 240 & \multirow{6}{*}{$\begin{array}{l}\text { Max } \\
\text { speed } \\
(\text { deg/sec) }\end{array}$} & 1 & 288 \\
\hline & & & & 2 & $-110+130$ & & 2 & 321 \\
\hline & & & & 3 & $-0+162$ & & 3 & 360 \\
\hline & & & & 4 & $+200-200$ & & 4 & 337 \\
\hline & & & & 5 & $-120+120$ & & 5 & 450 \\
\hline & & & & 6 & $-360+360$ & & 6 & 10977 \\
\hline
\end{tabular}
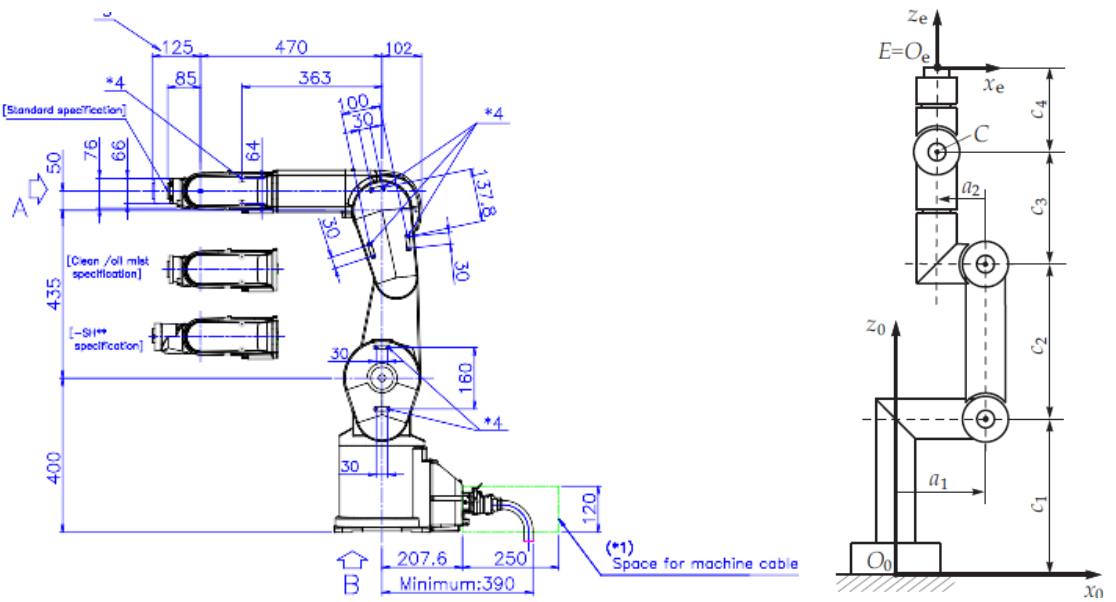

Figure 7

Mitsubishi RV-7FL external dimensions (left) and ortho-parallel basic and spherical wrist robot with seven essential geometric parameters [31]

Mitsubishi RV-7FL has a 3R ortho-parallel basis structure with a 3R wrist. Link and joint offsets were calculated and verified using a simulation toolbox. A summary of the kinematic analysis and the parameter values are shown in Table 2. 
Table 2

Structural Kinematic Parameters for Mitsubishi RV-7FL

\begin{tabular}{|c|c|c|c|}
\hline Joint No & Joint angles & Link Length $(\mathbf{m m})$ & Offsets $(\mathbf{m m})$ \\
\hline 1 & $\Theta_{1}$ & $\mathrm{~L}_{1}=\mathrm{c}_{1}=400$ & $\mathrm{a}_{1}=0$ \\
\hline 2 & $\Theta_{2}$ & $\mathrm{~L}_{2}=\mathrm{c}_{2}=435$ & $\mathrm{a}_{2}=-50$ \\
\hline 3 & $\Theta_{3}$ & $\mathrm{~L}_{3}=\mathrm{c}_{3}=470$ & $\mathrm{~b}=0$ \\
\hline 4 & $\Theta_{4}$ & $\mathrm{~L}_{4}=\mathrm{c}_{4}=85$ & \\
\hline 5 & $\Theta_{5}$ & 0 & \\
\hline 6 & $\Theta_{6}$ & 0 & \\
\hline
\end{tabular}

The forward and inverse kinematic problems were solved with Brandstötter, Angerer, and Hofbaur's algorithm [32] for Mitsubishi RV-7FL.

Transformation matrix of the end effector with respect to the base is given as:

$\boldsymbol{T}_{0}^{6}=\boldsymbol{T}_{0}^{1} \boldsymbol{T}_{1}^{2} \boldsymbol{T}_{2}^{3} \boldsymbol{T}_{3}^{4} \boldsymbol{T}_{4}^{5} \boldsymbol{T}_{5}^{6}=\left[\begin{array}{cccc} & & x(\boldsymbol{r p y}) & y \\ & & & z \\ 0 & 0 & 0 & 1\end{array}\right]$

The end effector position is given by:

$p=\left[\begin{array}{llllll}x & y & z & \theta_{x} & \theta_{y} & \theta_{z}\end{array}\right]^{T}$.

where

$$
\begin{aligned}
& \boldsymbol{T}_{0}^{6}(1,1)=s_{1}\left(c_{4} s_{6}+s_{4} c_{5} c_{6}\right)-c_{1}\left(c_{23}\left(s_{4} s_{6}-c_{4} c_{5} c_{6}\right)+s_{23} s_{5} c_{6}\right) \\
& \boldsymbol{T}_{0}^{6}(1,2)=s_{1}\left(c_{4} c_{6}+s_{4} c_{5} s_{6}\right)-c_{1}\left(c_{23}\left(s_{4} c_{6}+c_{4} c_{5} s_{6}\right)+s_{23} s_{5} s_{6}\right) \\
& \boldsymbol{T}_{0}^{6}(1,3)=c_{1}\left(s_{23} c_{5}+c_{23} c_{4} c_{5}\right)+s_{1} s_{4} s_{6} \\
& \boldsymbol{T}_{0}^{6}(1,4)=L_{4}\left(c_{1} s_{23} c_{5}+c_{1} c_{23} c_{4} s_{5}-s_{1} s_{4} s_{5}\right)+L_{3} c_{1} s_{23}-L_{2} c_{1} s_{2}-a_{2} c_{1} c_{23} \\
& \boldsymbol{T}_{0}^{6}(2,1)=c_{1}\left(c_{4} s_{6}+s_{4} c_{5} c_{6}\right)-s_{1}\left(c_{23}\left(s_{4} s_{6}-c_{4} c_{5} c_{6}\right)+s_{23} s_{5} c_{6}\right) \\
& \boldsymbol{T}_{0}^{6}(2,2)=c_{1}\left(c_{4} c_{6}+s_{4} c_{5} s_{6}\right)-s_{1}\left(c_{23}\left(s_{4} c_{6}+c_{4} c_{5} s_{6}\right)+s_{23} s_{5} s_{6}\right) \\
& \boldsymbol{T}_{0}^{6}(2,3)=s_{1}\left(s_{23} c_{5}+c_{23} c_{4} c_{5}\right)+c_{1} s_{4} s_{5} \\
& \boldsymbol{T}_{0}^{6}(2,4)=L_{4}\left(s_{1} s_{23} c_{5}+s_{1} c_{23} c_{4} s_{5}+c_{1} s_{4} s_{5}\right)+L_{3} c_{1} s_{23}+L_{2} s_{1} s_{2}+a_{2} s_{1} c_{23} \\
& \boldsymbol{T}_{0}^{6}(3,1)=s_{23}\left(s_{4} s_{6}-c_{4} c_{5} c_{6}\right)-c_{23} s_{5} c_{6} \\
& \boldsymbol{T}_{0}^{6}(3,2)=s_{23}\left(s_{4} c_{6}+c_{4} c_{5} s_{6}\right)+c_{23} s_{5} s_{6} \\
& \boldsymbol{T}_{0}^{6}(3,3)=\left(c_{23} c_{5}+s_{23} c_{4} s_{5}\right) \\
& \boldsymbol{T}_{0}^{6}(3,4)=L_{1}+L_{2} c_{2}+L_{4}\left(c_{23} s_{5}-s_{23} c_{4} s_{5}\right)+L_{3} c_{23}+a_{2} s_{1} c_{23}
\end{aligned}
$$

Wrist position can be calculated as:

$\left[\begin{array}{lll}x_{w} & y_{w} & z_{w}\end{array}\right]^{T}=\left[\begin{array}{lll}x & y & z\end{array}\right]^{T}-L_{4} R_{6}^{0}\left[\begin{array}{lll}0 & 0 & 1\end{array}\right]^{T}$ 


$$
\begin{aligned}
& \theta_{1}=\operatorname{atan} 2\left(y_{w}, x_{w}\right) \\
& \theta_{2}=\operatorname{acos}\left(\frac{M^{2}+L_{2}^{2}-K^{2}}{2 M L_{2}}\right)+\operatorname{atan} 2\left(N, z_{w}-L_{1}\right) \\
& \theta_{3}=\operatorname{acos}\left(\frac{M^{2}+L_{2}^{2}-K^{2}}{2 K L_{2}}\right)++\operatorname{atan} 2\left(a_{2}, L_{3}\right) \\
& \theta_{4}=\operatorname{atan} 2\left(\boldsymbol{T}_{0}^{6}(2,3) c_{1}-\boldsymbol{T}_{0}^{6}(1,3) s_{1}, \boldsymbol{T}_{0}^{6}(1,3) c_{23}+\boldsymbol{T}_{0}^{6}(2,3) c_{23} s_{1}-\boldsymbol{T}_{0}^{6}(3,3) s_{23}\right) \\
& \theta_{5}=\operatorname{atan} 2\left(\sqrt{1-H^{2}}, H\right) \\
& \theta_{6}=\operatorname{atan} 2\left(\boldsymbol{T}_{0}^{6}(1,2) s_{23} c_{1}-\boldsymbol{T}_{0}^{6}(2,2) s_{1} s_{23}+\boldsymbol{T}_{0}^{6}(3,2) c_{23,}-\boldsymbol{T}_{0}^{6}(1,1) s_{23} c_{1}\right. \\
& \left.\quad-\boldsymbol{T}_{0}^{6}(2,1) s_{23} s_{1}-\boldsymbol{T}_{0}^{6}(3,1) c_{23}\right)
\end{aligned}
$$

where

$$
\begin{aligned}
& N=\sqrt{x_{w}^{2}+y_{w}^{2}} \\
& M=\sqrt{N^{2}+\left(z_{w}^{2}-L_{1}\right)^{2}} \\
& K=\sqrt{a_{2}^{2}+L_{3}^{2}} \\
& H=\left(\boldsymbol{T}_{0}^{6}(1,3) s_{23} c_{1}+\boldsymbol{T}_{0}^{6}(2,3) s_{23} s_{1}-\boldsymbol{T}_{0}^{6}(3,3) c_{23}\right)
\end{aligned}
$$

A new Matlab-based program called LabanRobot was developed and used to write Labanotation for an industrial robot [33]. The user interface of LabanRobot is shown in Fig. 8. The choreographer can easily select the dance sequence, thinking about the geometry of the motion only, and imagining the robot as a human arm. The choreographer does not need to have any prior knowledge about the robot. They can design a dance using the interface and clicking on the rhythm and level boxes. The program plans the trajectory of the specified geometry.

The notation used in LabanRobot is different from traditional Labanotation. There are five levels, as an extra 'extension level' was added. The extension level, depending on the motion, is expressed with vertical or horizontal bars. Traditional Labanotation uses longer symbols for movements of longer duration, whereas LabanRobot does so with different colors. Rhythm is also represented by color. Using colors makes the script shorter.

The choreographer has to select the attributes of each movement: the rhythm (slow, medium, fast), the height or the level (low, medium-low, medium, mediumhigh, high), the extension (in, medium-in, medium, medium-out, out) and the direction. Next the movement is added to the dance sequence. The choreographer repeats this procedure for each movement in the sequence. Finally, the whole sequence is converted to a program by pressing the button 'Create Robot Program.' 


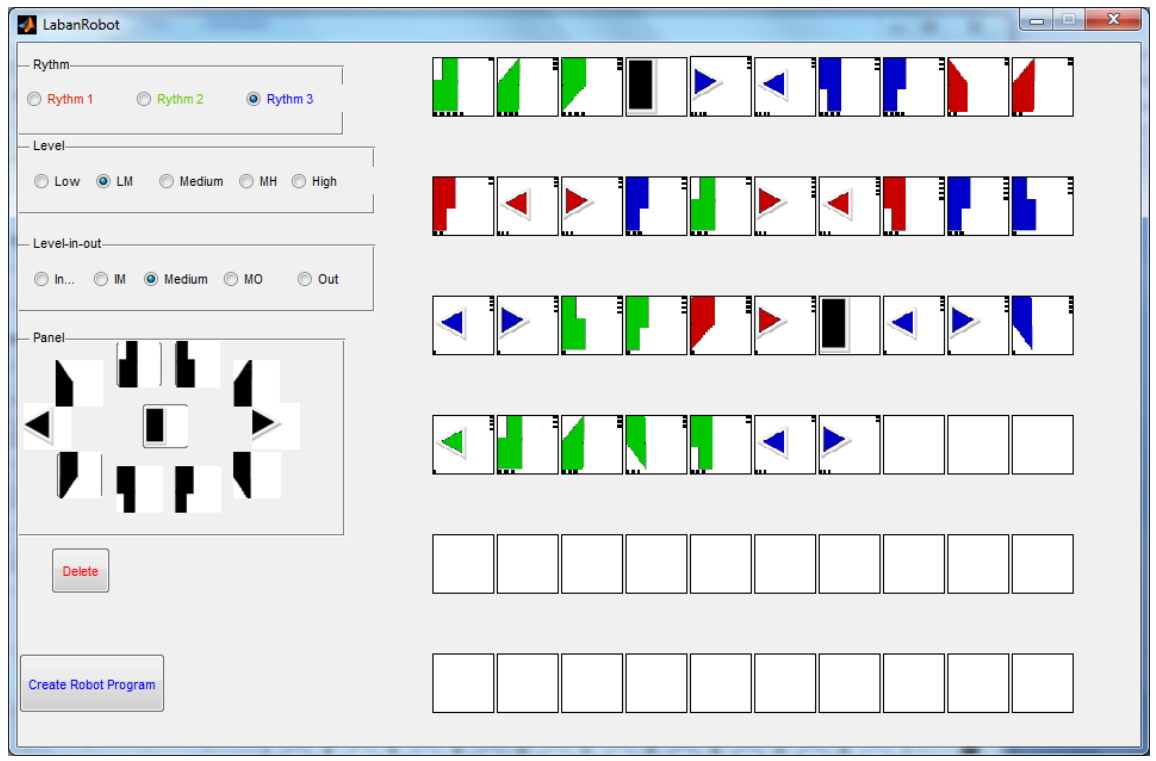

Figure 8

The user interface of LabanRobot

In Table 3, various heights and extensions are shown. The height of the end effector is designated by horizontal bars. The more bars, the higher the effector from the base. The extension is denoted by vertical bars. The more bars, the farther extended from the base.

The color codes indicate speed. Red is high speed, which is $70 \%$ of the robot's maximum acceleration. Green is medium speed, which is $40 \%$ of its maximum acceleration. Blue is low speed, which is $20 \%$ of its maximum acceleration.

Table 4 shows an example dance sequence to accompany the song Mi Chica sang by Sarbel [34]. The sequence consists of 12 steps. For each step, there are three rows. The first row shows the simulation result, the second shows the dance with the humans, and the third uses the extended Labanotation proposed in this paper.

\section{Conclusions}

An extended Labanotation for an industrial robot arm was developed herein. The notation is simpler than the original Labanotation, yet very effective. The height and the extension information were added to the traditional notation to increase smoothness and effect. The color codes were added to simplify notation of rhythm. The new program, LabanRobot, has a user-friendly and easy-to-use interface, where a choreographer can design a complete dance sequence with no prior knowledge of the robot. 
Table 3

Example for levels and extension levels

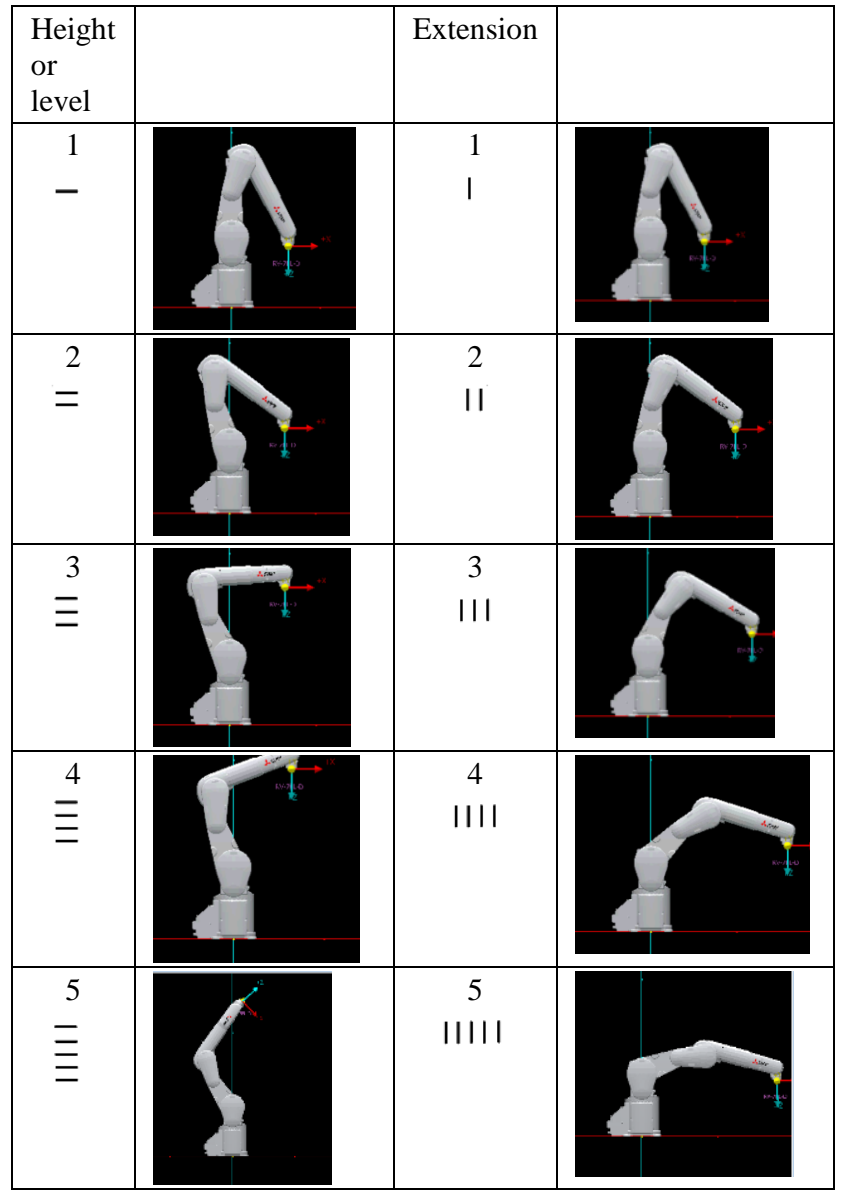

This research is unique in the sense that there is no other example of programming a dance for an industrial robot.

The algorithm was tested on an industrial robot on stage. The results were satisfactory. The next step of this research is to use the extended Labanotation on a multi-robot system and investigate human-robot interaction.

\section{Acknowledgement}

This work was supported by Mitsubishi Electric Turkey. 
Table 4

Dance sequence for RV-7FL robot with the Mi Chica song sang by Sabel

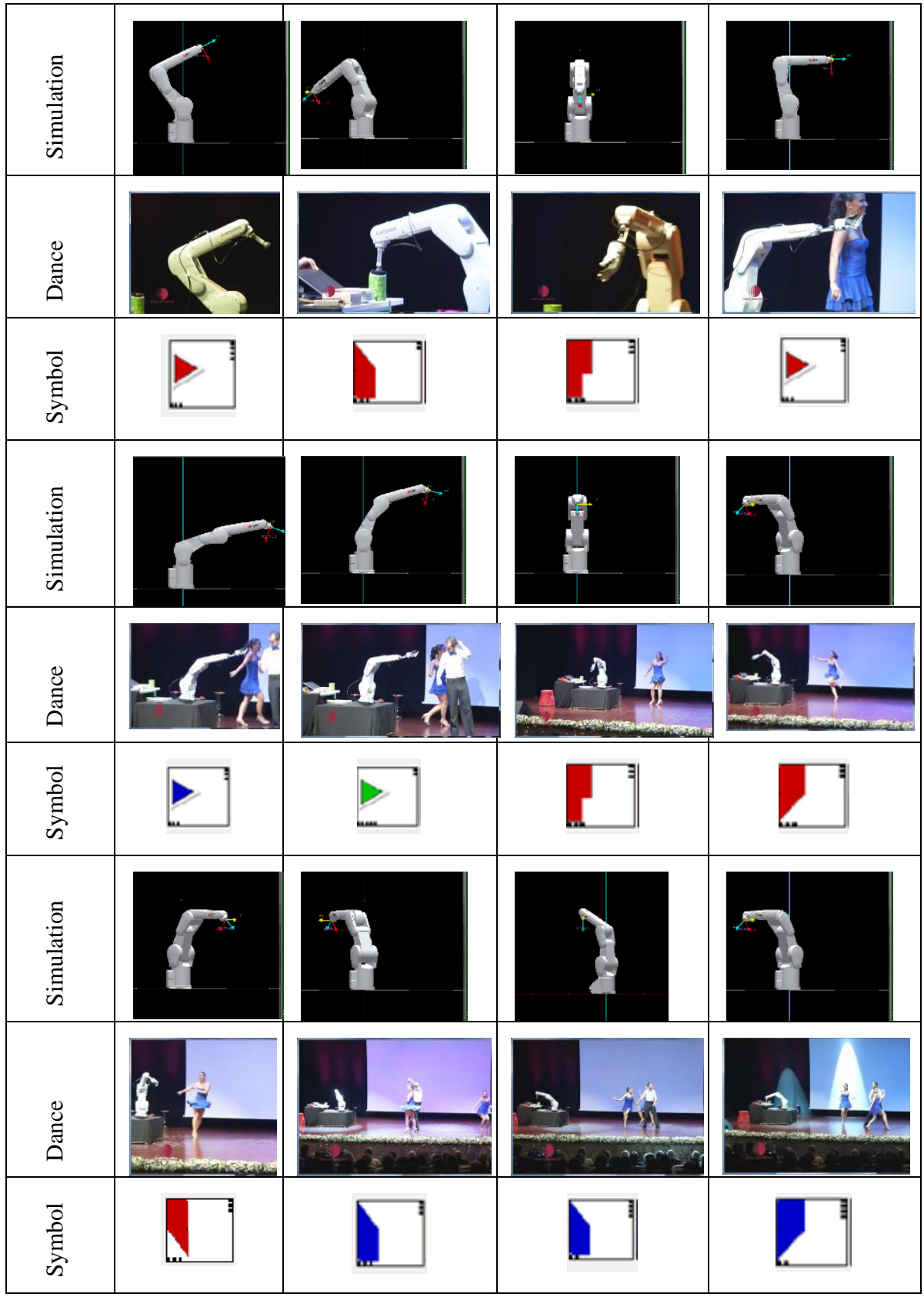




\section{References}

[1] E. Mirzabekiantz: Benesh Movement Notation for Humanoid Robots?, in Dance Notations and Robot Motion, Springer Tracts in Advanced Robotics, No. 111, J.-P. Laumond, N. Abe (eds), Switzerland, 2016, pp. 299-317

[2] J. Challet-Haas: The Problem of Recording Human Motion, in Dance Notations and Robot Motion, Springer Tracts in Advanced Robotics, No. 111, J.-P. Laumond, N. Abe (eds), Switzerland, 2016, pp. 69-89

[3] A. L. de Souza: Laban Movement Analysis-Scaffolding Human Movement, in Dance Notations and Robot Motion, Springer Tracts in Advanced Robotics, No. 111, J.-P. Laumond, N. Abe (eds), Switzerland, 2016, pp. 283-297

[4] S. J. Burton, A.-A. Samadani, R. Gorbet and D. Kulić: Laban Movement Analysis and Affective Movement Generation for Robots and Other NearLiving Creatures, in Dance Notations and Robot Motion, Springer Tracts in Advanced Robotics, No. 111, J.-P. Laumond, N. Abe (eds), Switzerland, 2016, pp. 25-48

[5] https://dance.osu.edu/research/dnb/laban-writer, accessed on March 10, 2016

[6] T. Calvert: Approaches to the Representation of Human Movement: Notation, Animation and Motion Capture, in Dance Notations and Robot Motion, Springer Tracts in Advanced Robotics, No. 111, J.-P. Laumond, N. Abe (eds), Switzerland, 2016, pp. 49-68

[7] A. La Viers, L. Bai, M. Bashiri, G. Heddy and Y. Sheng: Abstractions for Design-by-Humans of Heterogeneous Behaviours, in Dance Notations and Robot Motion, Springer Tracts in Advanced Robotics, No. 111, J.-P. Laumond, N. Abe (eds), Switzerland, 2016, pp. 237-262

[8] H. Drewes: MovEngine-Developing a Movement Language for 3D Visualization and Composition of Dance, in Dance Notations and Robot Motion, Springer Tracts in Advanced Robotics, No. 111, J.-P. Laumond, N. Abe (eds), Switzerland, 2016, pp. 91-116

[9] H. Peng, C. Zhou, H. Hu, F. Chao and J. Li: Robotic Dance in Social Robotics-A Taxonomy, IEEE Transactions on Human-Machine Systems, Vol. 45, No. 3, June 2015, pp. 281-293

[10] P. Afsar, P. Cortez and H. Santos: Automatic Visual Detection of Human Behavior: A Review from 2000 to 2014, Expert Systems with Applications, Elsevier, Vol. 42, 2015, pp. 6935-6956

[11] K. Hachimura, M. Nakamura, "Method of Generating Coded Description of Human Body Motion from Motion-captured Data," Proceedings of the IEEE International Workshop on Robot and Human Interactive Communication, 2001, pp. 122-127 
[12] K. Kojima, K. Hachimura and M. Nakamura: LabanEditor: Graphical Editor for Dance Notation, Proceedings of the 2002 IEEE International Workshop on Robot and Human Interactive Communication, Berlin, Germany, September 25-27, 2002, pp. 59-64

[13] A. Nakamura, T. Niwayama, S. Tabata and Y. Kumo: Development of a Basic Dance Training System with Mobile Robots, Proceedings of the 2004 IEEE International Workshop on Robot and Human Interactive Communication, Kurashiki, Okayama, Japan, September 20-22, 2004, pp. 211-216

[14] K. Suzuki and S. Hashimoto: Robotic Interface for Embodied Interaction via Dance and Musical Performance, Proceedings of the IEEE, Vol. 92, No. 4, April 2004, pp. 656-671

[15] K. Kosuge and Y. Hirata: Human-Robot Interaction, Proceedings of the 2004 IEEE International Conference on Robotics and Biomimetics, August 22-26, 2004, Shenyang, China, pp. 8-11

[16] Y. Choi, D. Kim, Y. Oh and B.-J. You: Posture/ Walking Control for Humanoid Robot Based on Kinematic Resolution of CoM Jacobian with Embedded Motion, IEEE Transactions on Robotics, Vol. 23, No. 6, December 2007, pp. 1285-1293

[17] I.-W. Jeong, Y.-H. Seo and H. S. Yang: Effective Humanoid Motion Generation based on Programming-by-Demonstration Method for Entertainment Robotics, Proceedings of the $16^{\text {th }}$ International Conference on Virtual Systems and Multimedia, Seoul, Korea, 2010, pp. 289-292

[18] Y. Kekehashi, T. Izawa, T. Shirai, Y. Nakanishi, K. Okada and M. Inaba: The Trials of Hula Hooping by a Musculo-Skeletal Humanoid KOJIRO Nearing Dancing Using the Soft Spine, Proceedings of the $11^{\text {th }}$ IEEE-RAS International Conference on Humanoid Robots, Bled, Slovenia, October 26-28, 2011, pp. 423-428

[19] X. Dong, K. Wang, G. Wang, L. Yan and I-M. Chen: Design and Study of a Highly Articulated Mini Lion-Dance Robot, IEEE/ASME International Conference on Advanced Intelligent Mechatronics, Montréal, Canada, July 6-9, 2010, pp. 830-835

[20] H. Wang, K. Kosuge: Control of a Robot Dancer for Enhancing Haptic Human-Robot Interaction in Waltz, IEEE Transactions on Haptics, Vol. 5, No. 3, July-September 2012, pp. 264-273

[21] F. Auguliaro, A. Schoellig and R. D'Andrea: Dance of the Flying Machines, IEEE Robotics \& Automation Magazine, December 2013, pp. 96-104

[22] F. Zhou, F. De la Torre and J. K. Hodgins: Hierarchical Aligned Cluster Analysis for Temporal Clustering of Human Motion, IEEE Transactions on 
Pattern Analysis and Machine Intelligence, Vol. 35, No. 3, March 2013, pp. $582-596$

[23] M.-B. Radac and R.-E. Precup: Optimal Behaviour Prediction Using a Primitive-Based Data-Driven Model-Free Iterative Learning Control Approach, Computers in Industry, Elsevier, Vol. 74, 2015, pp. 95-109

[24] H. Wang and Q. Zou: B-Spline-Decomposition-Based Approach to Multiaxis Trajectory Tracking: Nanomanipulation Example, IEEE Transactions on Control Systems Technology, Vol. 22, No. 4, July 2014, pp. $1573-1580$

[25] F. A. Mussa-Ivaldi and S. A. Solla: Neural Primitives for Motion Control, IEEE Journal of Oceanic Engineering, Vol. 29, No. 3, July 2004, pp. 640650

[26] D. J. Grymin, C. B. Neas and M. Farhood: A Hierarchical Approach for Primitive-Based Motion Planning and Control of Autonomous Vehicles, Robotics and Autonomous Systems, Elsevier, Vol. 62, 2014, pp. 214-228

[27] T. Okamoto, T. Shiratori, S. Kudoh, S. Nakaoka and K. Ikeuchi: Toward a Dancing RobotWith Listening Capability: Keypose-based Integration of Lower-, Middle-, and Upper-Body Motions for Varying Music Tempos, IEEE Transactions on Robotics, Vol 30, No. 3, June 2014, pp. 771-778

[28] O. E. Ramos, N. Mansard, O. Stasse, C. Benazeth, S. Hak and L. Saab: Dancing Humanoid Robots, IEEE Robotics \& Automation Magazine, December 2015, pp. 16-26

[29] S. Yoshida, T. Shirokura, Y. Sigiura, D. Sakamato, T. Ono, M. Inami and T. Igarashi: RoboJockey: Designing an Entertainment Experience with Robots, IEEE Computer Graphics and Applications, January/February 2016, 62-69

[30] W. Takano and Y. Nakamura: Real-time Unsupervised Segmentation of Human Whole-Body Motion and Its Application to Humanoid Robot Acquisition of Motion Symbols, Robotics and Automation Systems, Elsevier, Vol. 75, 2016, pp. 260-272

[31] Mitsubishi Industrial Robot CR750-D/CR751-D/CR760-D Controller RV4F-D/7F-D/13F-D/20F-D/35F-D/50F-D/70F-D Series Standard Specifications Manual, BFP-A8931-S, pp. 2-42, 2-44

[32] M. Brandstötter, A. Angerer and M. Hofbaur: An Analytical Solution of the Inverse Kinematics Problem of Industrial Serial Manipulators with an Ortho-parallel Basis and a Spherical Wrist, Proceedings of the Austrian Robotics Workshop, Linz, Austria, 22-23 May, 2014, pp. 7-11

[33] https://github.com/dtukel/RobotLaban

[34] https://www.youtube.com/watch?v=8rsR-hljltA 